\title{
Microvesicles as major biomarkers?
}

Identifying reliable biomarkers that can report disease presence, severity or progression is a major challenge for many tumour types. A new study describes a sensitive technique to detect and characterize glioblastomaderived microvesicles in the blood, as a potential diagnostic and monitoring tool for this disease.

Microvesicles are membranebound vesicles shed by numerous cell types that contain various

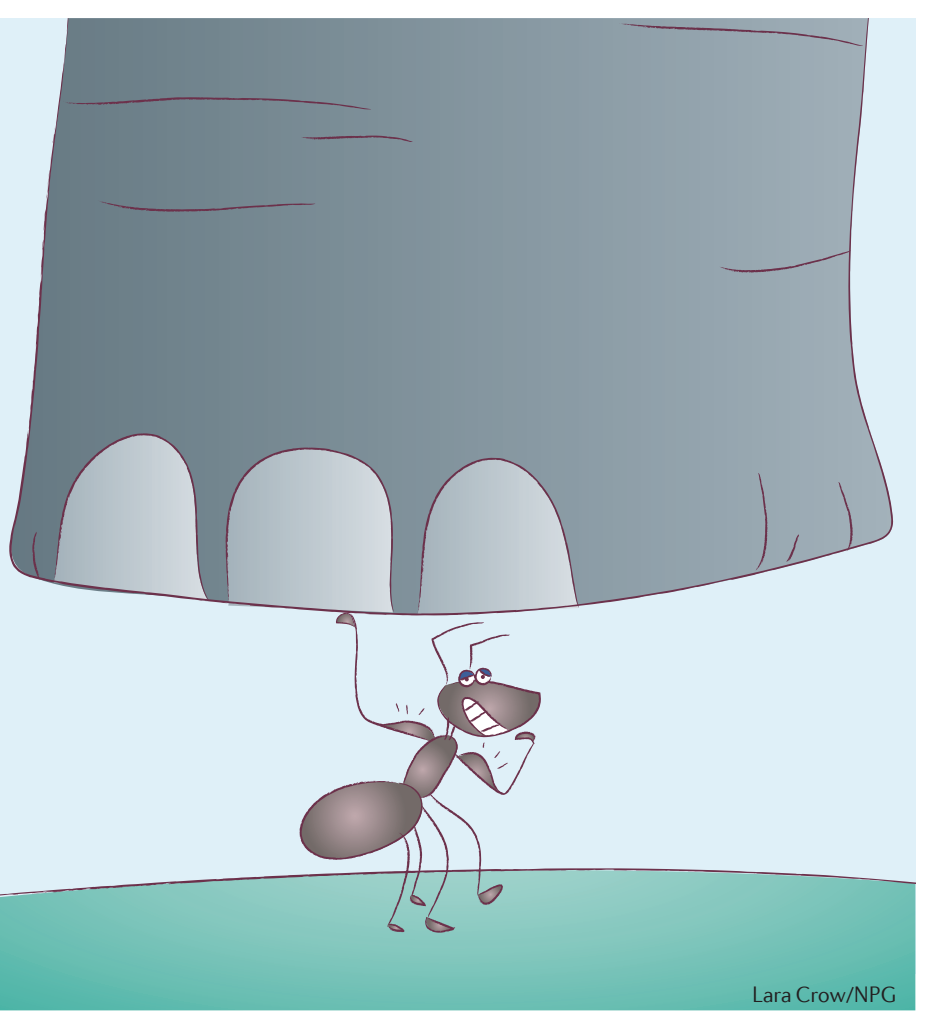

plasma membrane proteins (and other biomolecules) that are characteristic of the cell of origin. Hakho Lee, Ralph Weissleder and colleagues adapted a method of microvesicle detection that they had previously devised for whole-cell analysis. It involves immuno-magnetic labelling and micro-nuclear magnetic resonance $(\mu N M R)$ detection. For ease of clinical application, a microfluidic device was developed that can label microvesicles with antibody-bound magnetic nanoparticles (MNPs), filter out unbound antibodies or MNPs on the basis of particle size, and can detect labelled microvesicles using an integrated microcoil.

The authors tested this device on cell-culture-derived microvesicle preparations. A monoclonal antibody targeted to CD63 - which is found in microvesicles from various cell types - allowed the quantitation of total microvesicle numbers that was at least 100 -fold more sensitive than other detection methods, such as those based on western blotting or flow cytometry. Furthermore, the use of additional monoclonal antibodies enabled the quantification of the levels of proteins in the microvesicles, and this strongly corresponded to the expression levels of these proteins in the cell lines from which the nanoparticles were prepared.

Such analyses were also applied to microvesicle preparations from human blood. Crucially, the use of combinations of monoclonal antibodies targeted to both mutant and wild-type epidermal growth factor receptor (EGFR), wild-type podoplanin (PDPN) and mutant isocitrate dehydrogenase 2 (IDH2) could distinguish between samples from patients with glioblastoma and samples from healthy individuals with $90 \%$ accuracy, thus demonstrating the clinical diagnostic potential.

The technique was also used dynamically to monitor the response to temozolomide treatment of cultured glioblastoma cells, human glioblastoma xenografts in mice, and patients with glioblastoma. Decreases in glioblastoma cell numbers were reflected by a concomitant reduction in glioblastomaderived microvesicles in the tissue culture medium or in the blood. Interestingly, antitumour responses in mice and humans were seen more rapidly by microvesicle detection than by tumour imaging.

It will be interesting to determine the extent to which microvesicle analyses might supplement more traditional methods for clinical diagnosis and monitoring, and whether such technology can be applied to other tumour types.

Darren J. Burgess

ORIGINAL RESEARCH PAPER Shao, H. et al. Protein typing of circulating microvesicles allows real-time monitoring of glioblastoma therapy. Nature Med. 11 Nov 2012 (doi:10.1038/nm.2994) 